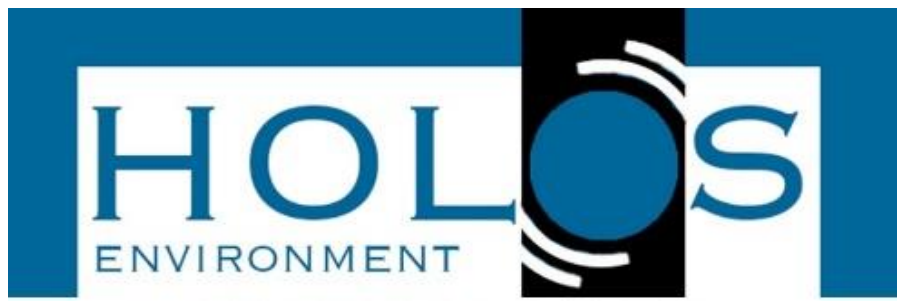

\title{
EFEITO DE DIFERENTES TAXAS DE AERAÇÃO NA REMOÇÃO DE NITROGÊNIO TOTAL E DQO EM ALAGADOS CONSTRUÍDOS DE FLUXO SUBSUPERFICIAL HORIZONTAL AERADOS INTERMITENTEMENTE
}

\section{EFFECT OF DIFFERENT AERATION RATES ON TOTAL NITROGEN AND COD REMOVAL IN INTERMITTENTLY AERATED HORIZONTAL SUBSURFACE FLOW CONSTRUCTED WETLANDS}

\author{
Gabriela Barbosa da Costa $^{1}$; Isabela Pires da Silva ${ }^{1}$; João Gabriel Thomaz Queluz; \\ Marcelo Loureiro Garcia ${ }^{1}$
}

Artigo recebido em: 28/10/2019 e aceito para publicação em: 21/11/2019.

DOI: http://dx.doi.org/10.14295/holos.v19i4.12358

Resumo: O objetivo do trabalho foi avaliar o efeito de diferentes taxas de aeração na remoção de demanda química de oxigênio (DQO) e nitrogênio total (NT) em alagados construídos de fluxo subsuperficial horizontal aerados intermitentemente. Foram estudados cinco alagados, sendo a dinâmica de aeração feita em 3 aerações diárias, com duração de uma hora cada, utilizando diferentes taxas de aeração: $0,2,5,10,20 \mathrm{~L} \mathrm{~min}^{-1}$. Os alagados receberam uma vazão de $8,7 \mathrm{~L} \mathrm{dia}^{-1}$ de água residuária doméstica sintética, resultando em tempo de detenção de 3 dias. De cada alagado construído foram avaliados os seguintes parâmetros: DQO, oxigênio dissolvido, pH, NT, nitrato, nitrito, condutividade elétrica, temperatura e vazão. Após uma análise aprofundada dos resultados junto à estatística, foi possível determinar que a aeração tem papel fundamental na melhora da remoção de NT e DQO nos sistemas. Entretanto, as diferentes taxas de aeração neste caso, não se mostraram decisivas para tal feito, e sim estatisticamente suficientes para um desempenho bom e similar. Com respeito às eficiências dos parâmetros, a DBO é $3 \%$ superior nos alagados construídos com aeração intermitente quando comparados ao sem aeração, numa porcentagem média $97,5 \%$ a $98,24 \%$ de eficiência nos sistemas aerados. Já o NT teve uma eficiência $30 \%$ superior nos alagados com aeração, atingindo uma média de $80 \%$ a $83,6 \%$ de eficiência de remoção

Palavras-chave: Alagados Construídos. Aeração intermitente. DQO. Nitrogênio total.

Abstract: The aim of the present research was to evaluate the effect of different aeration rates on chemical oxygen demand (COD) and total nitrogen (TN) removal in five constructed wetlands with horizontal subsurface flow, intermittently aerated for 1 hour three times a day. Each constructed wetland will be evaluated using different aeration rates: $0,2,5,10$ and $20 \mathrm{~L} \mathrm{~min}^{-1}$. Wetlands will receive a daily flow of $8.7 \mathrm{~L}$ of synthetic domestic wastewater, resulting in 3 days of hydraulic retention time. The following parameters will be evaluated: $\mathrm{COD}$, dissolved oxygen, $\mathrm{pH}, \mathrm{TN}$, nitrate, nitrite, electrical conductivity, temperature and flow rate. After an analysis of the results and statistic conclusions, it was possible to determine that aeration plays a main role in the improvement of nitrogen and COD removal in the systems. However, the different aeration rates in this case were not decisive for this purpose, but statistically enough for good and similar performance. Regarding the analysis of the efficiency of the parameters, the COD removal had a 3\% higher performance in the wetlands with intermittent aeration when compared to the non-aerated system, in a mean percentage of $97.5 \%$ to $98.24 \%$ efficiency. The

\footnotetext{
${ }^{1}$ Universidade Estadual Paulista (UNESP), Rio Claro, SP, Brasil. E-mails: (gabrielacosta-1997@hotmail.com, isabelapires@hotmail.com, i.queluz@unesp.br, marcelo.garcia@unesp.com)
} 
NT also had a $30 \%$ higher efficiency in the aerated wetlands, reaching an average of $80 \%$ to $83.6 \%$ of removal.

Keywords: Constructed wetlands. Intermittent aeration. COD. Total nitrogen.

\section{INTRODUÇÃO}

De acordo com o SNIS de 2015, somente $50,3 \%$ dos brasileiros possuem a rede coletora de esgoto e apenas $42,7 \%$ dos esgotos gerados tiveram tratamento adequado. Os índices, que não são satisfatórios, têm piores focos na zona rural do país. De acordo com a PNAD (IBGE, 2015) menos de $25 \%$ da população rural do Brasil teve acesso ao esgotamento sanitário. Apesar deste panorama, alguns processos, tais como as fossas sépticas, compostagens e sistemas alagados construídos são empregados como alternativas viáveis no tratamento de efluentes domésticos nas zonas rurais (KOBIYAMA; MOTA; CORCEUIL, 2008). Os alagados construídos (ACs), que são o foco da pesquisa em questão, segundo diversos estudos, mostram-se adequados a pequenas comunidades (LI et al., 2014; WU et al., 2014).

ACs com fluxo subsuperficial vertical e de fluxo horizontal, similarmente, apresentam um bom desempenho na remoção de demanda bioquímica de oxigênio (DBO), demanda química de oxigênio (DQO) e sólidos suspensos totais (SST), que são parâmetros essenciais na análise pós tratamento. Assim como os coliformes fecais e totais, cuja redução é significativa também nestes sistemas (ABOU-ELELA, 2012; CALIJURI et al., 2009). Entretanto, um dos parâmetros insatisfatórios é o desempenho na remoção de NT (VYMAZAL, 2007; WU et al., 2014).

Pesquisas indicam que uma maneira eficiente de se remover o NT da água é o sistema NDS (Nitrificação e Desnitrificação Simultâneas). A efetividade do processo depende crucialmente do oxigênio dissolvido (OD), pois os processos dominantes no sistema são definidos pela sua concentração na água (ZOPPAS; BERNARDES; MENEGUZZI, 2016). Entretanto os SACs falham na demanda de oxigênio (HU et al., 2012), pois as bactérias nitrificantes o disputam com a biodegradação orgânica. Especialmente em alagados de fluxo subsuperficial, esta falha limita a nitrificação e consequentemente a remoção de NT (FAN et al., 2013).

Desta forma, a aeração artificial vem sendo investigada por diversos pesquisadores como uma solução (WU et al., 2014), pois remove o NT de forma mais eficiente comparado aos alagados construídos tradicionais (FAN et al., 2013; FOLADORI et al., 
2013; NIVALA et al., 2007; WU et al., 2016; ZOPPAS; BERNARDES; MENEGUZZI, 2016).

Atualmente, não é encontrada uma pesquisa exclusiva de aeração intermitente que compara taxas de oxigênio, com intuito de definir a quantidade que proporciona uma performance ideal em alagados construídos de fluxo horizontal subsuperficial. Desta forma, o objetivo da presente pesquisa foi analisar o efeito de diferentes taxas de aeração na remoção de DQO e NT em alagados construídos de fluxo subsuperficial horizontal com aeração intermitente.

\section{MATERIAL E MÉTODOS}

O experimento foi conduzido em ambiente protegido (estufa agrícola) no Centro de Estudos Ambientais (CEA) - UNESP, Rio Claro, SP. O clima da região é definido como Cwa (Koppën): clima temperado quente (mesotérmico) com verão chuvoso e inverno seco, a temperatura média do mês mais quente supera $22^{\circ} \mathrm{C}$. A precipitação e temperatura médias anuais são respectivamente, $1366,8 \mathrm{~mm}$ e $21,6^{\circ} \mathrm{C}$.

\subsection{Características dos sistemas de tratamento}

Cinco ACs de fluxo subsuperficial horizontal em escala piloto constituíram o sistema em questão. Cada AC era composto por uma caixa d'água retangular de polipropileno com capacidade aproximada de 61 litros. Como meio suporte utilizou-se brita \#0, com porosidade média de 53\%, determinada em laboratório com o uso de proveta graduada de um litro e água destilada. O meio suporte preencheu cada caixa até a altura de $30 \mathrm{~cm}$ e ficou saturado pelo efluente até $25 \mathrm{~cm}$. Assim, conhecendo-se as dimensões das caixas d'água, a altura do nível do efluente e a porosidade do meio, calculou-se que cada $\mathrm{AC}$ apresentava volume útil aproximado de 26 litros. Os alagados construídos são mostrados na Figura 1. 
Figura 1 - (A) alagados construídos no dia do plantio das macrófitas (07/03/2018); e (B) alagados construídos sete meses após o plantio das macrófitas (12/10/2018)

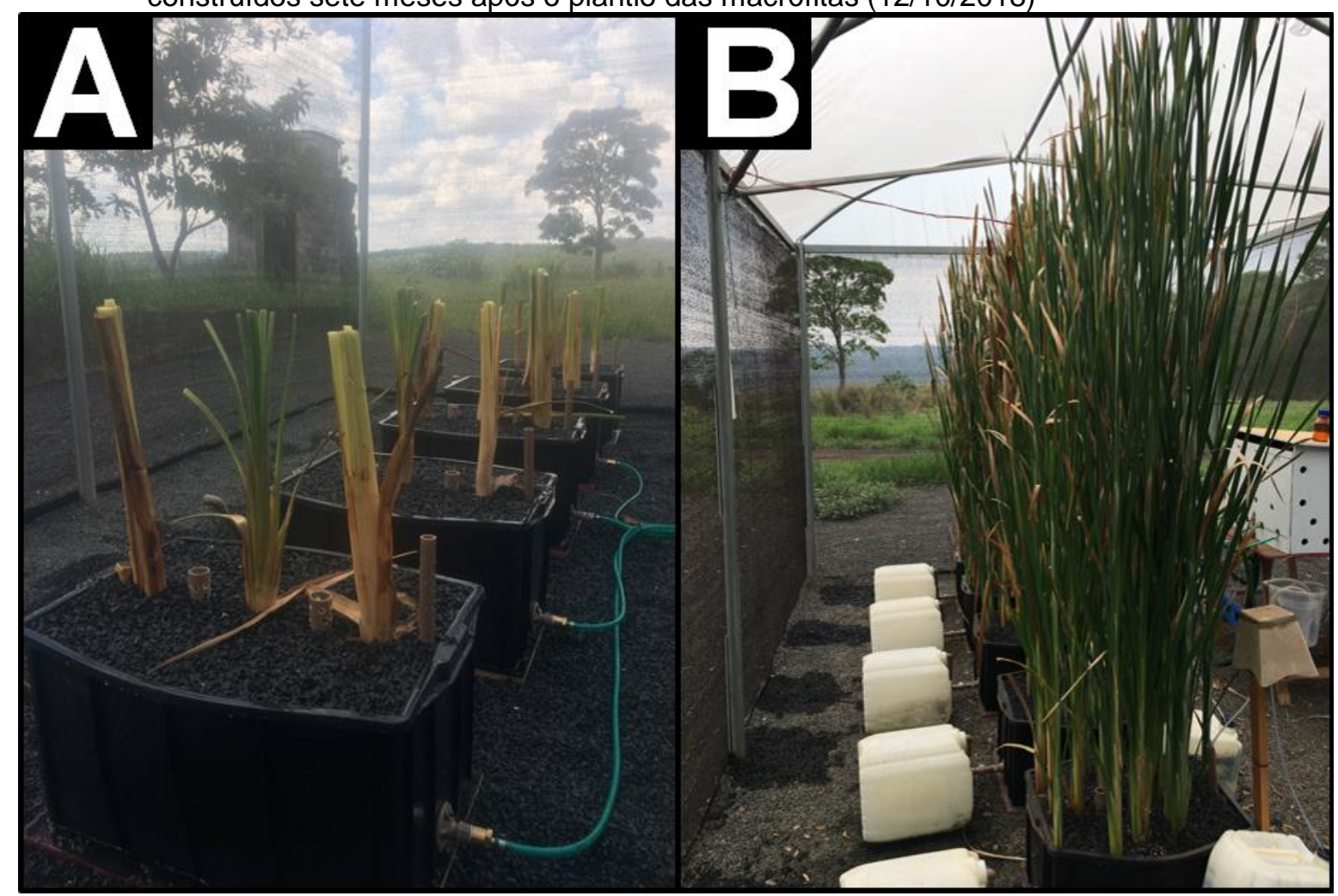

A macrófita emergente utilizada nos sistemas foi a Typha latifólia, popularmente conhecida como Taboa, coletada em brejo natural e podada à meia altura, afim de manter a capacidade fotossintética da planta. O replantio nos alagados construídos foi realizado no dia 07/03/2018 com propágulos constituídos de plantas completas (rizoma + parte aérea). A densidade aproximada de plantio foi de 15 plantas por $\mathrm{m}^{2}$, ou seja, cada alagado construído foi cultivado inicialmente com três plantas. Após a fixação da espécie vegetal, iniciou-se a aplicação de água residuária, no dia 02/04/2018.

\subsection{Origem do afluente e operação do sistema}

O experimento foi conduzido utilizando água residuária sintética, que apresentava características físico-químicas semelhantes à de efluente primário de tanque séptico, ou seja, este efluente não apresentava sólidos ou gorduras. O efluente sintético era preparado utilizando água de abastecimento com a adição de $0,1880 \mathrm{~g}$ $\mathrm{L}^{-1}$ de $\left(\mathrm{NH}_{4}\right)_{2} \mathrm{SO}_{4}, 0,0180 \mathrm{~g} \mathrm{~L}^{-1}$ de $\mathrm{KH}_{2} \mathrm{PO}_{4}, 0,0205 \mathrm{~g} \mathrm{~L}^{-1}$ de $\mathrm{MgSO}_{4} .7 \mathrm{H} 2 \mathrm{O}, 0,0183 \mathrm{~g} \mathrm{~L}^{-}$ ${ }^{1}$ de $\mathrm{FeSO}_{4} .7 \mathrm{H} 2 \mathrm{O}, 0,0133 \mathrm{~g} \mathrm{~L}^{-1}$ de $\mathrm{CaCl}_{2} .2 \mathrm{H} 2 \mathrm{O}$ e $0,3860 \mathrm{~g} \mathrm{~L}^{-1}$ de sacarose, sendo que 
esta era adicionada somente no preparo do efluente sintético. A composição do efluente sintético é descrita nos trabalhos de Fan et al. (2016), Wu et al. (2015) e Wu et al. (2016).

Por meio de bombas peristálticas, eram aplicados $8,7 \mathrm{~L} \mathrm{~d}^{-1}$ do efluente sintético em cada um dos cinco ACs. Assim, sabendo-se que o volume útil de cada SAC era de aproximadamente 26 litros, determinou-se que o tempo de detenção hidráulica nominal era de 3 dias. Transcorridos os dois dias, todos os reservatórios de entrada eram limpos e novamente reabastecidos.

\subsection{Aeração intermitente}

Os ACs eram aerados intermitentemente em 3 períodos com duração de 1 hora (1 $\mathrm{h}$ aerando/7 h sem aeração) distribuídos igualmente ao longo do dia. Assim, visando avaliar o efeito de diferentes aerações na remoção de poluentes em alagados construídos, os sistemas eram operados com diferentes taxas de aplicação de oxigênio: $A C$ 1: sem aeração $0 \mathrm{~L} \mathrm{~min}-1$; $A C$ 2: $2 \mathrm{~L} \mathrm{~min}{ }^{-1}$; $A C$ 3: $5 \mathrm{~L} \mathrm{~min}{ }^{-1}$; $A C$ 4: $10 \mathrm{~L}$ $\mathrm{min}^{-1}$; e AC 5: $20 \mathrm{~L} \mathrm{~min}{ }^{-1}$. As diferentes vazões eram obtidas com 0 uso de compressores de ar para aquário disponíveis no mercado. O oxigênio era aplicado nos alagados construídos com um difusor tubular de ar (mangueira porosa para piscicultura) instalado no fundo e ao longo da seção longitudinal das caixas de polipropileno (Figura 2). Os compressores de ar de foram controlados (ligados e desligados) automaticamente utilizando temporizador de tomada digital.

\subsection{Coletas, análises e monitoramento do sistema}

Foram realizadas cinco baterias de coleta de amostras nos dias 01/11/2018, 09/11/2018, 13/11/2018, 23/11/2018 e 07/12/2018. Os parâmatros avaliados nas zonas de entrada e saída dos alagados construídos foram: DQO, pH, NT, nitrato, nitrito, condutividade elétrica, temperatura e vazão. As amostras foram coletadas, preservadas e analisadas físicoquimicamente de acordo com os métodos definidos pelo Standard Methods (APHA, 2012).

Além disso, a vazão efluente foi medida com o auxílio de reservatórios de $20 \mathrm{~L}$ padrão Inmetro (similar ao reservatório auxiliar) acoplados ao tubo de saída efluente 
de cada AC. A medição do volume do efluente coletado era feita com o advento de recipientes graduados e provetas.

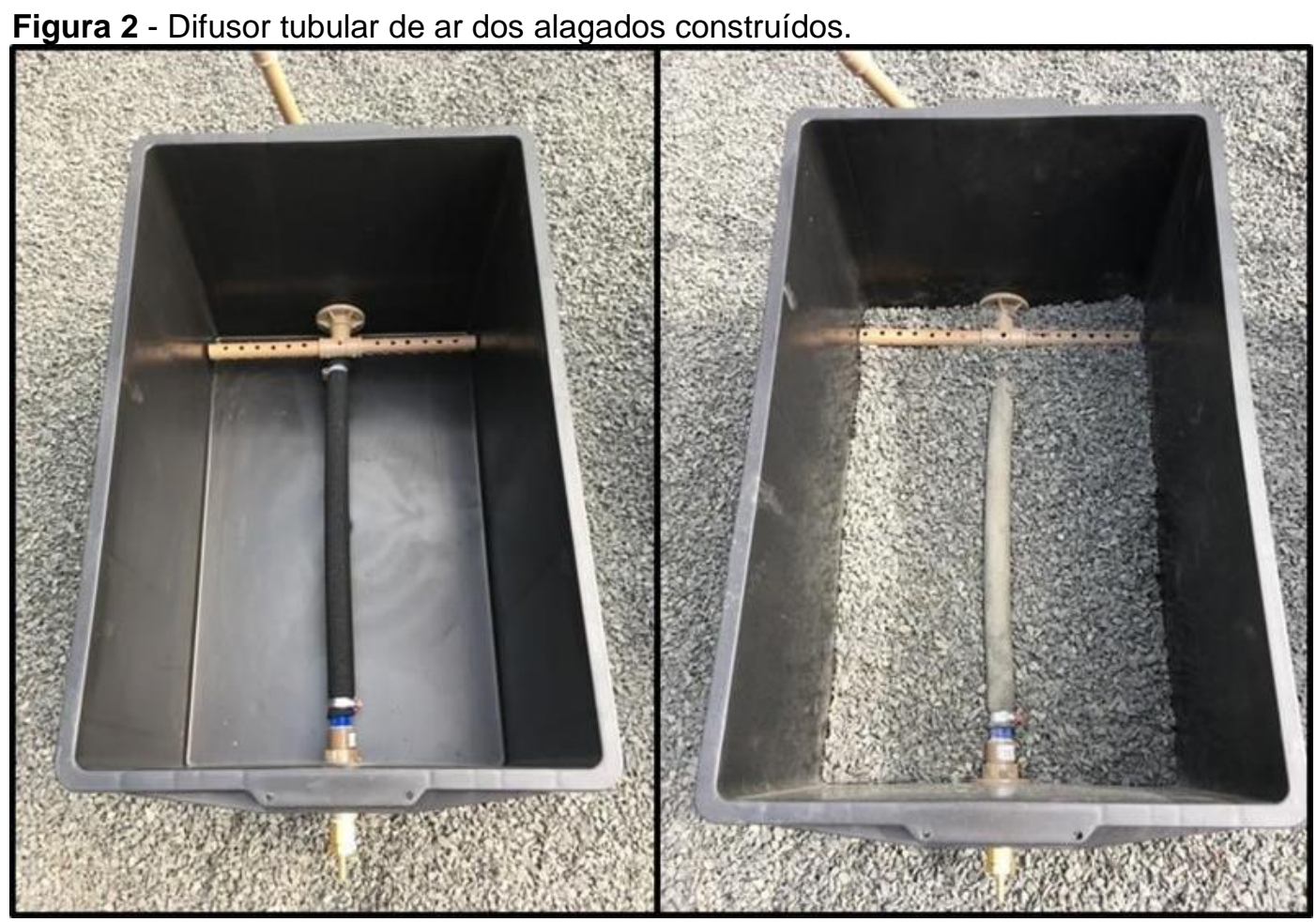

\subsection{Análise estatística}

Os dados de DQO e NT obtidos nos ACFHs foram comparados por análise de variância (ANOVA) e a descriminação entre as médias foi realizada pelo teste de Tukey. As análises foram realizadas no programa estatístico Statgraphics (versão 16. 2. 04) e para ambos os testes (ANOVA e Tukey) foi adotado nível de significância de $5 \%(P<0,05)$.

\section{RESULTADOS E DISCUSSÃO}

Após terem sido finalizadas as etapas do projeto, foram analisados todos os resultados e formulados os tópicos a serem discutidos. Sendo estes, a análise físicoquímica, a análise estatística e a análise de eficiência dos sistemas. Com todas essas etapas cumpridas, foi possível determinar qual é a taxa de aeração que propicia uma melhor eficiência do tratamento nos sistemas alagados construídos. 


\subsection{Desempenho geral dos alagados constuídos}

Na Tabela 1 são mostradas as características físico-químicas do afluente e dos efluentes dos cinco sistemas estudados à respeito de todos os parâmetros analisados na pesquisa. Os valores foram obtidos realizando a média dos parâmetros nas 5 baterias de análise.

Tabela 1 - Valores médios das características físico-químicas do afluente e efluente dos alagados

\begin{tabular}{|c|c|c|c|c|c|c|}
\hline \multirow{2}{*}{ Parâmetro } & \multirow{2}{*}{ Afluente } & \multicolumn{5}{|c|}{ Efluente alagados construídos } \\
\hline & & AC1 & AC2 & AC3 & AC4 & AC5 \\
\hline $\mathrm{DQO}\left(\mathrm{mg} \mathrm{L}^{-1}\right)$ & $388,8 \pm 19,7$ & $23,8 \pm 6,6$ & $9,6 \pm 5,1$ & $8,2 \pm 3,7$ & $9,4 \pm 4,3$ & $6,8 \pm 2,9$ \\
\hline $\mathrm{NT}\left(\mathrm{mg} \mathrm{L}^{-1}\right)$ & $40,5 \pm 2,3$ & $20,8 \pm 2,0$ & $8,0 \pm 2,6$ & $7,9 \pm 1,5$ & $7,6 \pm 1,5$ & $6,6 \pm 1,2$ \\
\hline $\mathrm{NH}_{4}{ }^{+}\left(\mathrm{mg} \mathrm{L}^{-1}\right)$ & $39,2 \pm 2,3$ & $16,5 \pm 1,0$ & $0,8 \pm 0,8$ & $0,6 \pm 0,6$ & $0,5 \pm 0,5$ & $0,6 \pm 0,3$ \\
\hline $\mathrm{NO}_{3}^{-}\left(\mathrm{mg} \mathrm{L}^{-1}\right)$ & $1,4 \pm 0,3$ & $4,3 \pm 1,6$ & $7,0 \pm 3,0$ & $7,2 \pm 1,3$ & $7,0 \pm 1,3$ & $6,0 \pm 1,1$ \\
\hline $\mathrm{NO}_{2}^{-}\left(\mathrm{mg} \mathrm{L}^{-1}\right)$ & $\begin{array}{c}0,004 \pm \\
0,002\end{array}$ & $\begin{array}{c}0,02 \pm \\
0,01\end{array}$ & $0,09 \pm 0,18$ & $0,10 \pm 0,14$ & $0,05 \pm 0,07$ & $0,05 \pm 0,08$ \\
\hline $\mathrm{PO}_{4}{ }^{3-}\left(\mathrm{mg} \mathrm{L}^{-1}\right)$ & $3,9 \pm 0,5$ & $1,6 \pm 0,7$ & $0,9 \pm 0,6$ & $0,9 \pm 0,5$ & $1,2 \pm 0,7$ & $0,9 \pm 0,7$ \\
\hline $\mathrm{OD}\left(\mathrm{mg} \mathrm{L}^{-1}\right)$ & - & $0,5 \pm 0,2$ & $1,4 \pm 0,6$ & $2,0 \pm 1,3$ & $2,4 \pm 1,6$ & $3,0 \pm 2,1$ \\
\hline $\mathrm{pH}$ & $7,0 \pm 0,3$ & $7,1 \pm 0,4$ & $6,7 \pm 0,5$ & $6,5 \pm 0,6$ & $6,3 \pm 0,6$ & $6,7 \pm 0,5$ \\
\hline $\mathrm{CE}\left(\mu \mathrm{S} \mathrm{cm} \mathrm{cm}^{-1}\right)$ & $564 \pm 18$ & $397 \pm 16$ & $439 \pm 41$ & $474 \pm 25$ & $441 \pm 33$ & $579 \pm 73$ \\
\hline $\mathrm{T}\left({ }^{\circ} \mathrm{C}\right)$ & $23.1 \pm 1,5$ & $21,5 \pm 1,7$ & $20,8 \pm 0,8$ & $20,1 \pm 1,4$ & $20,2 \pm 1,8$ & $20,3 \pm 1,8$ \\
\hline
\end{tabular}

\subsection{Remoção da demanda química de oxigênio}

A seguir, na Figura 3 e na Tabela 2, são mostrados o comportamento e as remoções de DQO para os cinco alagados construídos durante a execução do experimento, respectivamente.

A análise dos resultados mostra uma sutil melhora de remoção de DQO nos sistemas aerados, sendo aproximadamente 4\% maior quando comparada à eficiência geral de um sistema não aerado. No sistema sem aeração (AC1), a eficiência média gira em torno de 93,9\%, com $23,8 \mathrm{mg} \mathrm{L}^{-1}$ de DQO remanescente, enquanto nos sistemas de aeração intermitente essa porcentagem média de eficiência vale de 97,5 (AC2) a 98,3\% (AC5) e suas respectivas DQOs remanescentes equivalem a 9,6 e 6,8 $\mathrm{mg} \mathrm{L}^{-1}$. 
Figura 3 - Remoção de DQO para os alagados construídos.

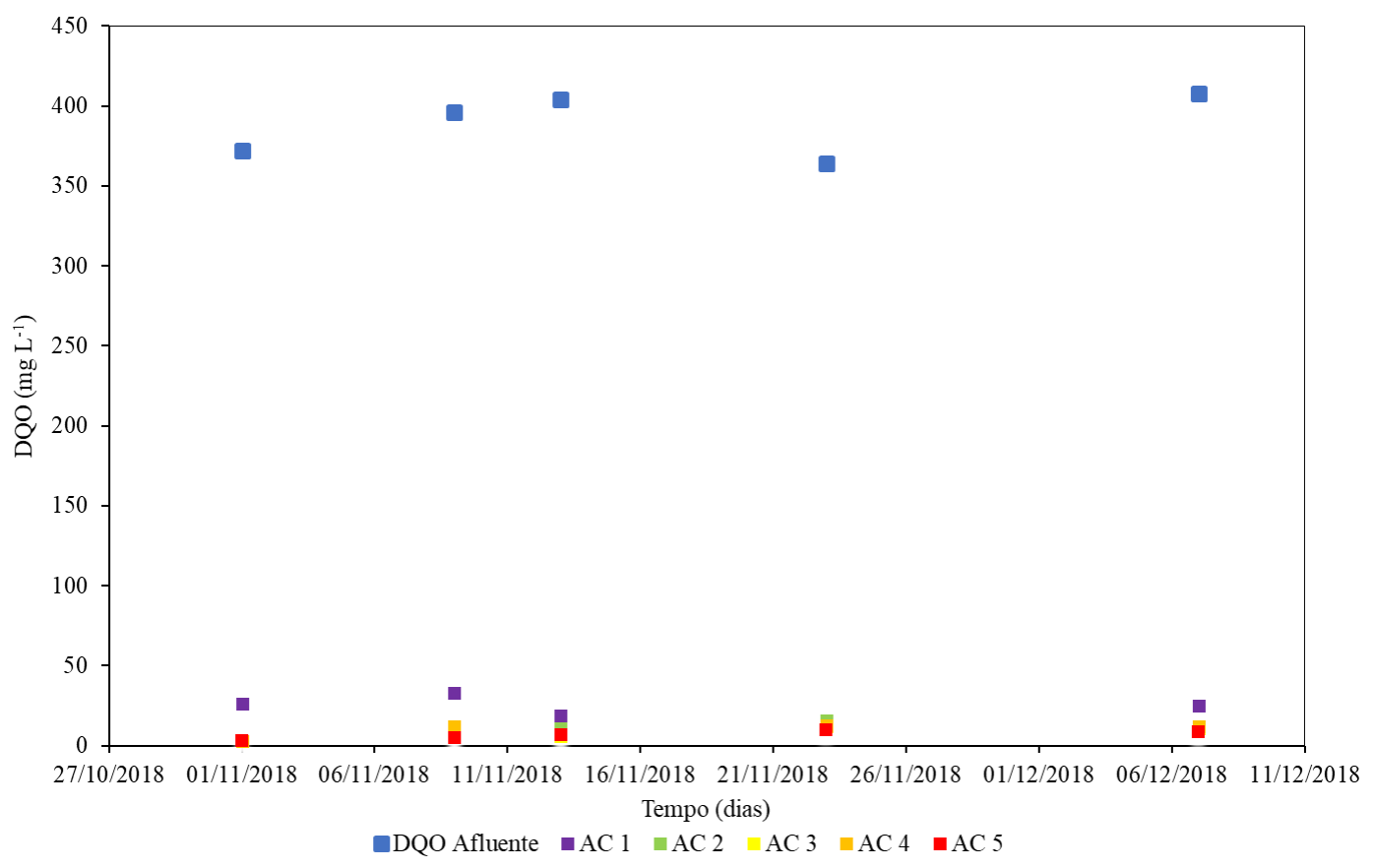

Tabela 2 - Demanda química do afluente e efluente dos alagados construídos e suas respectivas taxas de remoção

DQO Valor máximo Valor mínimo

$\left(\mathrm{mg} \mathrm{L}^{-1}\right)$

$\left(\mathrm{mg} \mathrm{L}^{-1}\right)$

$\left(\mathrm{mg} \mathrm{L}^{-1}\right)$

$\begin{array}{ccccc}\text { Afluente } & 388,8 \pm 19,7 & 408 & 364 & \\ \text { AC 1 } & 23,8 \pm 6,6 & 33 & 16 & 93,9 \\ \text { AC 2 } & 9,6 \pm 5,1 & 16 & 3 & 97,5 \\ \text { AC 3 } & 8,2 \pm 3,7 & 12 & 3 & 97,9 \\ \text { AC4 } & 9,4 \pm 4,3 & 13 & 3 & 97,6 \\ \text { AC 5 } & 6,8 \pm 2,9 & 10 & 3 & 98,3\end{array}$

Essa melhora se deve aos mecanismos de remoção inerentes aos sistemas com os ambientes aeróbios e anaeróbios alternados, onde respectivamente as bactérias aeróbias e anaeróbias degradam a matéria orgânica. Nos sistemas aerados, predomina a remoção de material orgânico carbonáceo através da respiração aeróbia, enquanto em condições anaeróbias e anóxicas os mecanismos são diversos e incluem a fermentação e a desnitrificação (QUELUZ, 2016).

Os resultados encontrados neste trabalho assemelham-se aos de outras publicações com renome. Wu et al. (2016) atingiu 95\% de eficiência em remoção de DQO em alagados construídos de fluxo subsuperficial com aeração intermitentemente para tratamento de esgoto doméstico. Eficiências de $97,2 \%$ e $96 \%$ em DQO também foram 
obtidas (WU et al., 2016; WU et al., 2014). Em Zhang (2016), 95,6\% de remoção de DQO em ACs de parâmetros similares em fluxo e aeração.

Existem, na literatura, trabalhos onde a eficiência mostra-se inferior quando comparada ao presente trabalho, como em Yusoff et al. (2018), que obteve redução de 109,9 para $38,9 \mathrm{mg} \mathrm{L}^{-1}$ de DQO, representando $66,1 \%$ de remoção em alagados construídos de fluxo subsuperficial, cultivado com S. Grossus, para efluentes de fábrica de celulose e reciclagem de papel. Assim como o percentual de remoção de $69,9 \%$ e 64,8\% com $P$. Australis e $C$. Papyrus, com águas residuárias com tratamento primário em alagados construídos em escala piloto de fluxo subsuperficial vertical, na pesquisa de García-Ávila et al. (2019). Essa distinção, possivelmente, se da em decorrência da diferente origem dos efluentes.

Tendo em base os resultados obtidos neste trabalho e apresentados na Tabela 2, apesar dos sistemas aerados não apresentarem uma diferença substancial em relação à eficiência de remoção, o AC 5 apresentou uma melhor performance. Sua taxa de aeração injetava $20 \mathrm{~L} \mathrm{~min}^{-1}$, sendo a maior taxa do experimento. Sua DQO média equivale a $6,8 \mathrm{mg} \mathrm{L}^{-1}$, e possui a menor variância dentre todos os sistemas, ou seja, durante a bateria de análises, foi o sistema que apresentou a menor discrepância e oscilação em seus valores, mantendo uma eficiência de remoção consistente.

\subsection{Remoção de nitrogênio amoniacal}

Na Figura 4 e na Tabela 3, são mostrados o comportamento e as remoções de $\mathrm{NH}_{4}{ }^{+}$para os cinco alagados construídos, de acordo com as análises realizadas, respectivamente.

Os resultados obtidos na análise mostram uma melhora acentuada na remoção de $\mathrm{NH}_{4}{ }^{+}$nos sistemas aerados, elevando a eficiência em aproximadamente $40 \%$ quando comparada à eficiência geral de um sistema não aerado. No sistema sem aeração (AC1), a eficiência média gira em torno de $57,7 \%$, com $16,5 \mathrm{mg} \mathrm{L}^{-1}$ de $\mathrm{NH}_{4}{ }^{+}$ remanescente, enquanto nos sistemas de aeração intermitente essa porcentagem média de eficiência vale de 97,8\% (AC2) a 98,6\% (AC4) e suas respectivas $\mathrm{NH}_{4}{ }^{+}$remanescentes equivalem a 0,8 e $0,5 \mathrm{mg} \mathrm{L}^{-1}$. Observa-se um comportamento estável quanto à remoção de nitrogênio amoniacal ao comparar somente os alagados com diferentes taxas de aeração entre si. Assim, é possível inferir que até a menor taxa de 
aeração é suficiente para a obtenção de um bom resultado na remoção de $\mathrm{NH}_{4}{ }^{+}$nos sistemas.

Figura 4 - Remoção de nitrogênio amoniacal para os alagados construídos

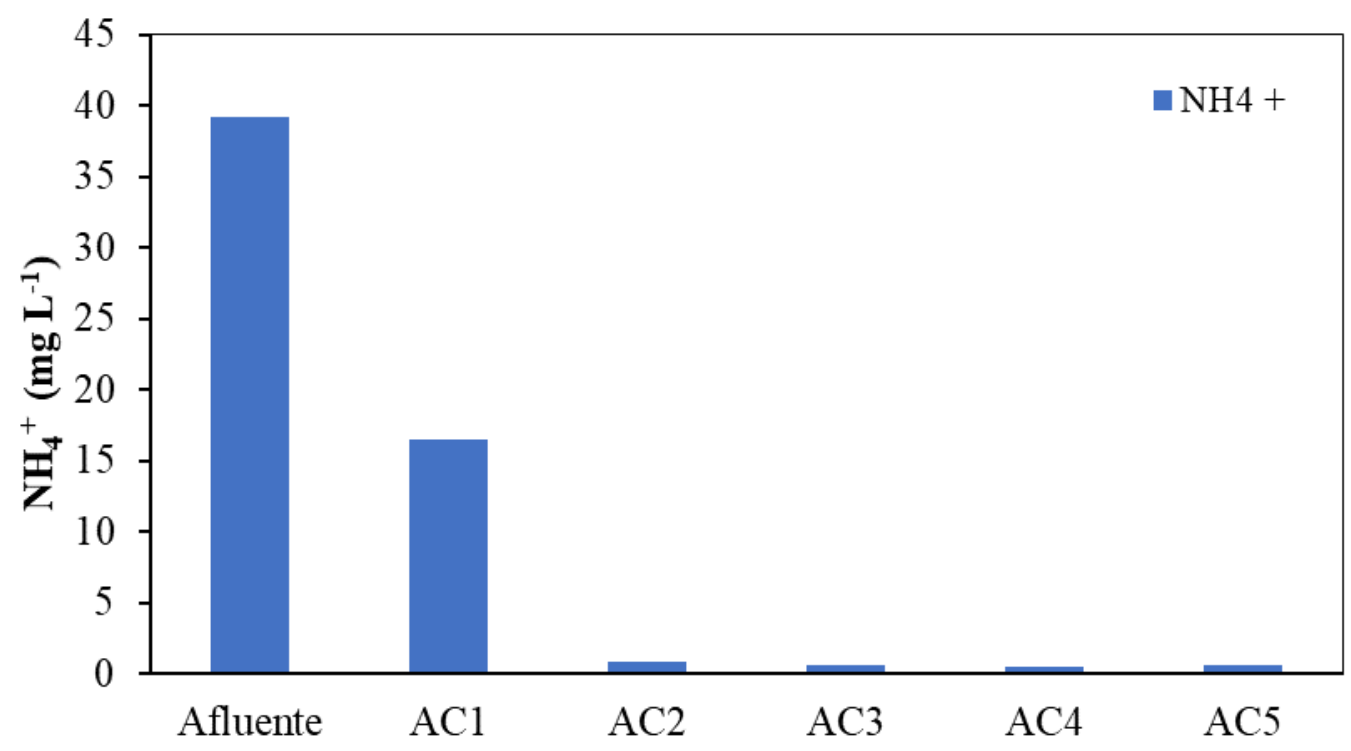

Tabela 3 - Remoção de nitrogênio amoniacal afluente e efluente para os sistemas

\begin{tabular}{ccccc}
\hline & $\begin{array}{c}\mathbf{N H}_{\mathbf{4}}{ }^{+} \\
\left(\mathbf{m g ~ L}^{-1}\right)\end{array}$ & $\begin{array}{c}\text { Valor máximo } \\
\left(\mathbf{m g ~ L}^{-1}\right)\end{array}$ & $\begin{array}{c}\text { Valor mínimo } \\
\left(\mathbf{m g ~ L}^{-1}\right)\end{array}$ & (\%) \\
\hline Afluente & $39,2 \pm 2,3$ & 42,9 & 36,7 & \\
AC 1 & $16,5 \pm 1,0$ & 17,6 & 15,3 & 57,7 \\
AC 2 & $0,8 \pm 0,8$ & 2,2 & 0,3 & 97,8 \\
AC 3 & $0,6 \pm 0,6$ & 1,7 & 0,3 & 98,4 \\
AC4 & $0,5 \pm 0,5$ & 1,5 & 0,3 & 98,6 \\
AC 5 & $0,6 \pm 0,3$ & 0,9 & 0,3 & 98,5 \\
\hline
\end{tabular}

A melhora na eficiência do sistema controle (AC1) quando comparado aos demais, se deve à disponibilidade de oxigênio, proporcionada pelo advento da aeração artificial. Em ambientes aeróbios, os sistemas realizam o processo de nitrificação, com as bactérias aeróbias, convertendo a amônia em nitrito e nitrato.

Existem na literatura, trabalhos com resultados similares aos obtidos pela presente pesquisa. Alguns destes mostram, em alagados construídos de fluxo vertical com aeração intermitente, remoção de amônia à 98,4\% (Wu et al., 2016a), 96,1\% na eficiência de remoção por Fan et al. (2016), assim como em 97,4\% e 98,8\% de eficiência em alagados construídos por Wu et al. (2016). 


\subsection{Remoção de nitrogênio total}

Foi investigada a remoção de nitrogênio total durante a execução do trabalho, e os resultados obtidos são complementados pela Figura 5 e pela Tabela 4.

Figura 5 - Remoção de nitrogênio total para os alagados construídos

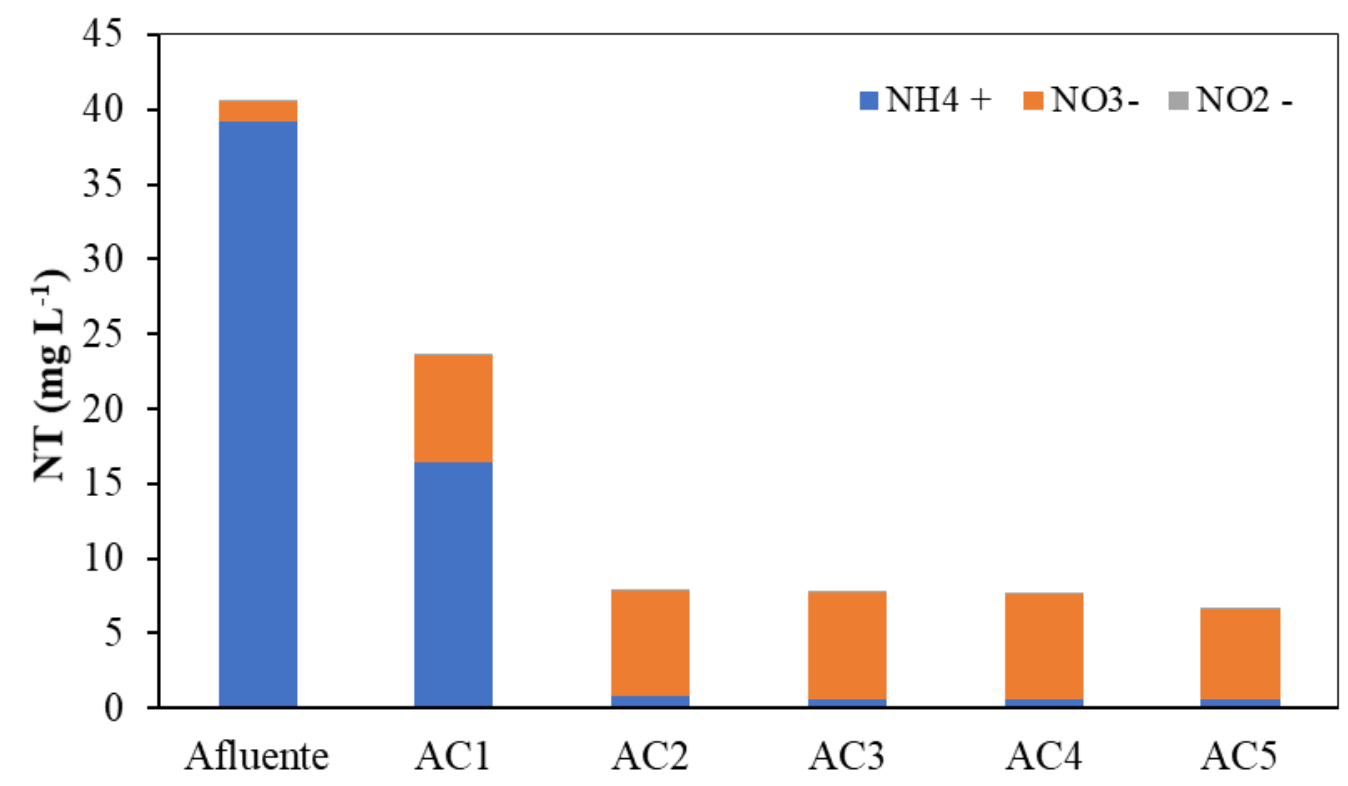

Tabela 4 - Remoção de nitrogênio total afluente e efluente para os sistemas

\begin{tabular}{ccccc}
\hline & $\begin{array}{c}\mathrm{NT} \\
\left(\mathrm{mg} \mathrm{L}^{-1}\right)\end{array}$ & $\begin{array}{c}\text { Valor máximo } \\
\left(\mathrm{mg} \mathrm{L}^{-1}\right)\end{array}$ & $\begin{array}{c}\text { Valor mínimo } \\
\left(\mathrm{mg} \mathrm{L}^{-1}\right)\end{array}$ & $(\%)$ \\
\hline Afluente & $40,5 \pm 2,3$ & 44,5 & 38,4 & \\
AC 1 & $20,8 \pm 2,0$ & 23,2 & 17,6 & 48,6 \\
AC 2 & $8,0 \pm 2,6$ & 11,9 & 4,5 & 80 \\
AC 3 & $7,9 \pm 1,5$ & 9,1 & 6,0 & 80,5 \\
AC4 & $7,6 \pm 1,5$ & 9,2 & 5,5 & 81 \\
AC 5 & $6,6 \pm 1,2$ & 8,4 & 5,3 & 83,6 \\
\hline
\end{tabular}

Segundo os dados obtidos e mostrados na tabela acima, nota-se um aumento de aproximadamente $30 \%$ na eficiência de remoção média de NT dos sistemas aerados intermitentemente. No sistema sem aeração (AC1), a eficiência média gira em torno de 48,67\%, com 20,8 $\mathrm{mg} \mathrm{L}^{-1}$ de NT remanescente, enquanto nos sistemas de aeração intermitente essa porcentagem média de eficiência vale de 80\% (AC2) a 
83,6\% (AC5) e seu respectivos NT remanescentes equivalem a 8,0 e 6,6 $\mathrm{mg} \mathrm{L}^{-1}$. Os dados produzidos concordam com a bibliografia existente em demais trabalhos, como o 85,8\% de eficiência por Fan et al. (2016) e 90,6\% por Wu et al. (2016a), ambos em alagados construídos com aeração intermitente.

Em relação ao nitrogênio total, o mecanismo responsável pela melhora de aproximadamente $30 \%$ na eficiência de tratamento, é a atuação da nitrificação e desnitrificação simultâneas, proporcionada pela aeração intermitente nos sistemas.

Em todos os sistemas, nota-se uma quantidade ínfima de $\mathrm{NO}_{2}^{-}$, e este fato nos permite dizer que a transformação de nitrito para nitrato se dava de forma rápida. $O$ efluente do $\mathrm{AC} 1$ era preponderantemente composto por $\mathrm{NH}^{+}$, devido à nitrificação limitada. Tal fator se deve à ausência de oxigênio, pois o tratamento foi realizado em ambiente anaeróbio. A porcentagem média de remoção foi de $48,6 \%$ enquanto nos sistemas aerados atingiu entre $80 \%$ e $83,6 \%$. Os demais sistemas, eram compostos majoritariamente por $\mathrm{NO}_{3}^{-}$, indicando que houve nitrificação efetiva. Este sistema age nestas duas interfaces, onde o nitrogênio amoniacal se converte em nitrito e nitrato nos ambientes aeróbios, em seguida, em ambientes anaeróbios o nitrato se converte em nitrogênio gasoso (ZOPPAS; BERNARDES; MENEGUZZI, 2016). Ademais, é necessário que se mencione o papel da absorção pela macrófita para a remoção do NT, pois os sistemas aerados são abuntantes em nitrato. Segundo Vymazal (2007), em ambientes ricos neste ânion, este torna-se a fonte mais importante de nitrogênio nutriente para o crescimento da planta, através de suas raízes ou até mesmo brotos de plantas submersos, contribuindo para a eficiência de sua remoção na água.

É importante ressaltar também que, após a estabilização do sistema alagado, um acúmulo de nitrato nos sistemas com aeração intermitente é notório na análise final de eficiência. Este se deve, possivelmente, pela falta de carbono orgânico no meio e à diminuição da desnitrificação pela predominância do ambiente aerado e (MALTAIS-LANDRY et al., 2009). Podemos constatar a falta de carbono orgânico no meio com o auxílio da Tabela 2 e da Figura 4, onde os dados de DQO dos alagados são citados, estando abaixo de $10 \mathrm{mg} \mathrm{L}^{-1}$ nos sistemas aerados. Assim, a desnitrificação nestes sistemas não pode ser realizada completamente. 


\subsection{Análise estatística}

A Tabela 5 a seguir mostra a análise de variância (ANOVA) para os parâmetros de DQO e NT dos experimentos.

Tabela 5 - ANOVA dos parâmetros DQO e NT obtidos nos cinco alagados construídos na segunda etapa do experimento

\begin{tabular}{ccccccc}
\hline \multirow{2}{*}{ Parâmetro } & \multicolumn{5}{c}{ ANOVA } \\
\cline { 2 - 7 } & Fonte & SQ & GL & QM & $\begin{array}{c}\text { F- } \\
\text { Teste }\end{array}$ & P-Valor \\
\hline \multirow{2}{*}{ DQO } & Entre grupos & 961,36 & 4 & 240,34 & 10,90 & $<\mathbf{0 , 0 0 0 1}$ \\
& Dentro de grupos & 440,8 & 20 & 22,04 & & \\
& Total (Corr.) & 1402,16 & 24 & & & \\
\hline \multirow{2}{*}{ NT } & Entre grupos & 706,75 & 4 & 176,688 & 51,98 & $<\mathbf{0 , 0 0 0 0}$ \\
& Dentro de grupos & 67,988 & 20 & 3,4 & & \\
& Total (Corr.) & 774,739 & 24 & & & \\
\hline
\end{tabular}

GL: Grau de Liberdade; SQ: Soma dos Quadrados; QM: Quadrado Médio

Através do ANOVA, a variância foi decomposta em intergrupos (entre os alagados construídos) e intragupos (para cada alagado construído), para os parâmetros de DQO e NT, afim de originar o índice F. O índice F, equivale a 10,90 para DQO e 51,98 para NT. Ele é calculado pela razão entre a estimativa intergrupos e intragrupos. Como o valor de $\mathrm{P}$ é menor que 0,05 para ambos os casos, sabe-se que existe uma diferença estatística significativa entre as médias de DQO e NT de um alagado construído para outro no nível de significância de 5\%. Entretanto, para que sejam determinadas quais médias são significativamente diferentes das outras, foi realizado o Teste de Tukey.

Os resultados obtidos no Teste de Tukey para os parâmetros DQO e NT mostram que há diferença estatística significativa entre o alagado construído sem aeração e os demais sistemas, separando-os em diferentes grupos de homogeneidade. $\mathrm{O}$ alagado sem aeração obteve uma média de 23,8 e 20,76 para DQO e NT, respectivamente. Para o outro grupo de homogeneidade (alagados aerados) as médias variam entre 6,8 e 9,6 para DQO e 6,61 e 7,97 para NT, indicando que não há diferença significativa entre os sistemas aerados a $5 \%$ de probabilidade. 


\section{CONCLUSÕES}

Com respeito aos parâmetros físico-químicos, conclui-se que tanto na DQO quanto no NT, os sistemas aerados intermitentemente mostraram um melhor desempenho quando comparados ao alagado não aerado. As porcentagens obtidas para DQO e NT em alagados não aerados foram de $93,9 \%$ e $48,6 \%$, enquanto em alagados com aeração chegaram em até e $98,3 \%$ e 83,6\%, com melhorias de $30 \%$ e $3 \%$ aproximadamente. Assim é comprovada importância do sistema de aeração no tratamento realizado por alagados construídos de fluxo subsuperficial horizontal.

Finalmente, conclui-se que a falta de aeração nos sistemas alagados minimiza seu potencial para a remoção de NT e DQO, entretanto, todas as taxas propostas de aeração melhoram seu desempenho, sem existir uma a se ressaltar como melhor.

\section{AGRADECIMENTOS}

Agradecemos às agências de fomento FAPESP (processo FAPESP 2017/18075-8) e CNPQ (processo PIBIC 2018/046830) por viabilizar a pesquisa, e ao Centro de Estudos Ambientais (CEA-UNESP) por disponibilizar os laboratórios e a área experimental.

\section{REFERÊNCIAS}

ABOU-ELELA, S.; GOLINELLI, G; EL-TABL, A.; HELLAL, M. Treatment of municipal wastewater using horizontal flow constructed wetlands in Egypt. Water Science and Technology, v. 69, n. 1, p.38-47, 2013.

APHA - American Public Health Association. Standard methods for the examination for water and wastewater. 22. ed. Washington, Dc: APHA-AWWA-WEF, 2012. 1496 p.

CALIJURI, M. L.; BASTOS, R. K. X.; MAGALHÃES, T. B.; CAPELETE, B. C.; DIAS, E. H. O. Tratamento de esgotos sanitários em sistemas reatores UASB/wetlands construídas de fluxo horizontal: eficiência e estabilidade de remoção de matéria orgânica, sólidos, nutrientes e coliformes. Engenharia Sanitaria e Ambiental, v. 14, n. 3, p.421-430, 2009.

CHEN, Y.; WEN, Y.; ZHOU, Q.; VYMAZAL, J. Effects of plant biomass on nitrogen transformation in subsurface batch constructed wetlands: A stable isotope and mass balance assessment. Water Research, v. 63, p.158-167, 2014.

FAN, J; ZHANG, B; ZHANG, J; NGO, H.H.; GUO, W; LIU, F; GUO, Y; WU, H. Intermittent aeration strategy to enhance organics and nitrogen removal in subsurface flow constructed wetlands. Bioresource Technology, v. 141, p.117-122, 2013. 
FAN, J.; ZHANG, J.; GUO, W.; LIANG, S.; WU, H. Enhanced long-term organics and nitrogen removal and associated microbial community in intermittently aerated subsurface flow constructed wetlands. Bioresource Technology, v. 214, p.871-875, 2016.

FOLADORI, P.; RUABEN, J.; ORTIGARA, A. R. Recirculation or artificial aeration in vertical flow constructed wetlands: A comparative study for treating high load wastewater. Bioresource Technology, v. 149, p.398-405, 2013.

GARCÍA-ÁVILA, F.; PATIÑO-CHÁVEZ, J.; ZHINÍN-CHIMBO, F.; DONOSO-MOSCOSO, S.; FLORES DEL PINO, L.; AVILÉS-AÑAZCO, A. Performance of Phragmites Australis and Cyperus Papyrus in the treatment of municipal wastewater by vertical flow subsurface constructed wetlands. International Soil and Water Conservation Research, v. 7, n. 3, p.286296, set. 2019.

HU, Y.; ZHAO, Y.; ZHAO, X.; KUMAR, J. G. High Rate Nitrogen Removal in an Alum SludgeBased Intermittent Aeration Constructed Wetland. Environmental Science \& Technology, v. 46, n. 8, p.4583-4590, 2012.

Instituto Brasileiro de Geografia e Estatística - IBGE. Pesquisa Nacional do Saneamento Básico de 2008. Rio de Janeiro: MCIDADES, 2010. 219 p. Disponível em: https://biblioteca.ibge.gov.br/visualizacao/livros/liv45351.pdf. Acesso em: 23 out. 2019.

KANTAWANICHKUL, S.; KLADPRASERT, S.; BRIX, H. Treatment of high-strength wastewater in tropical vertical flow constructed wetlands planted with Typha angustifolia and Cyperus involucratus. Ecological Engineering, v. 35, n. 2, p.238-247, 2009.

KOBIYAMA, M.; MOTA, A. A.; CORCEUIL, C. W. Saneamento rural. In: Seminário Saneamento Ambiental. Rio Negrinho: ACIRNE, Anais, 2008. 24p. Disponível em: http://www.labhidro.ufsc.br/Proje-

tos/ARTI 2008/Artigo\%202\%20 Kobiyama\%20Mota\%20e\%20Corceuil .pdf. Acesso em: 24 out. 2019 .

LI, F.; LU, L.; ZHENG, X.; ZHANG, X. Three-stage horizontal subsurface flow constructed wetlands for organics and nitrogen removal: Effect of aeration. Ecological Engineering, $v$. 68, p.90-96, 2014.

LISBOA, S. S.; HELLER, L.; SILVEIRA, R. B. Desafios do planejamento municipal de saneamento básico em municípios de pequeno porte: a percepção dos gestores. Engenharia Sanitaria e Ambiental, v. 18, n. 4, p.341-348, dez. 2013.

MALTAIS-LANDRY, G.; MARANGER, R.; BRISSON, J.; CHAZARENC, F. Nitrogen transformations and retention in planted and artificially aerated constructed wetlands. Water Research, v. 43, n. 2, p.535-545, 2009.

YUSOFF, M. F. M.; ABDULLAH, S. R. S.; HASAN, H. A. Performance of continuous pilot subsurface constructed wetland using Scirpus grossus for removal of COD, colour and suspended solid in recycled pulp and paper effluent. Environmental Technology \& Innovation, v. 13, p. 346-352, 2019.

NASCIMENTO, T. L. Estudo do uso de Wetlands Construídas no tratamento de esgotos domésticos em comunidades rurais. 70f. Monografia (Graduação) - Escola de Engenharia de Lorena, Universidade de São Paulo, Lorena, 2015. 
NIVALA, J.; HOOS, M. B.; CROSS, C.; WALLACE, S.; PARKIN, G. Treatment of landfill leachate using an aerated, horizontal subsurface-flow constructed wetland. Science Of The Total Environment, v. 380, n. 1-3, p.19-27, 2007.

QUELUZ, J. G. T. Eficiência de alagados construídos para o tratamento de águas residuárias com baixas cargas orgânicas. 2016. 110 f. Tese (Doutorado) - Curso de Agronomia (Irrigação e Drenagem), Universidade Estadual Paulista, Faculdade de Ciências Agronômicas, Botucatu, 2016.

Sistema nacional de informações sobre saneamento - SNIS. Diagnóstico dos Serviços de Água e Esgotos. Brasília: SNSA/MCIDADES, 2015. 212 p. p. 26. Disponível em: http://www.snis.gov.br/diagnostico-agua-e-esgotos/diagnostico-ae-2015.

VYMAZAL, J. Removal of nutrients in various types of constructed wetlands. Science Of The Total Environment, v. 380, n. 1-3, p.48-65, 2007.

VYMAZAL, J. Constructed wetlands, surface flow. In: JØRGENSEN, S.E.; FATH, B. (Eds.) Encyclopedia of Ecology. Amsterdam: Elsevier BV, 2008. v 1.

VYMAZAL, J.; KRÖPFELOVÁ, L. Wastewater Treatment in Constructed Wetlands with Horizontal Sub-Surface Flow. Environmental Pollution, Dordrecht, p.1-10, 2008. Springer Netherlands. http://dx.doi.org/10.1007/978-1-4020-8580-2.

WANG, W.; DING, Y.; WANG, Y.; SONG, X.; AMBROSE, R. F.; ULLMAN, J. L. Intensified nitrogen removal in immobilized nitrifier enhanced constructed wetlands with external carbon addition. Bioresource Technology, v. 218, p.1261-1265, 2016.

WANG, W.; DING, Y.; WANG, Y.; SONG, X.; AMBROSE, R. F.; ULLMAN, J. L.; WINFREY, B. K.; WANG, J.; GONG, J. Treatment of rich ammonia nitrogen wastewater with polyvinyl alcohol immobilized nitrifier biofortified constructed wetlands. Ecological Engineering, v. 94, p.7-11, 2016.

WU, S.; KUSCHK, P.; BRIX, H.; VYMAZAL, J.; DONG, R. Development of constructed wetlands in performance intensifications for wastewater treatment: A nitrogen and organic matter targeted review. Water Research, v. 57, p.40-55, jun. 2014. Elsevier BV.

WU, H.; FAN, J.; ZHANG, J.; NGO, H.H.; GUO, W.; HU, Z.; LIANG, S. Decentralized domestic wastewater treatment using intermittently aerated vertical flow constructed wetlands: impact of influent strengths. Bioresource Technology, v. 176, p.163-168. 2015

Wu, H.; Fan, J.; Zhang, J.; Ngo, H.H.; Guo, W.; Liang, S; Lv, J.; Lu, S.; Wu, W.; Wu, S. (2016) Intensified organics and nitrogen removal in the intermittent-aerated constructed wetland using a novel sludge-ceramsite as substrate. Bioresource Technology, v. 210, p. 101107,2016

WU, H.; FAN, J.; ZHANG, J.; NGO, H. H.; GUO, W.; HU, Z.; LV, J. Optimization of organics and nitrogen removal in intermittently aerated vertical flow constructed wetlands: Effects of aeration time and aeration rate. International Biodeterioration \& Biodegradation, v. 113, p.139-145, 2016a.

ZOPPAS, F. M.; BERNARDES, A. M.; MENEGUZZI, A. Parâmetros operacionais na remoção biológica de nitrogênio de águas por nitrificação e desnitrificação simultânea. Engenharia Sanitária e Ambiental, v. 21, n. 1, p.29-42, 2016. 